\title{
Career Advancement: The Experiences of Minority Nurses in Accessing Leadership Positions in a Tertiary Care Setting
}

\author{
Naima Bouabdillah (P.I.) ${ }^{1}$, Amélie Perron ${ }^{2} \&$ Dave Holmes $^{2}$ \\ ${ }^{1}$ Departement of Nursing, Université du Québec à Trois Rivières, Québec, Canada. \\ ${ }^{2}$ School of Nursing, University of Ottawa, Ontario, Canada. \\ Cite as: Bouabdillah, N., Perron, A., \& Holmes, D. (2021). Career Advancement: The Experiences of \\ Minority Nurses in Accessing Leadership Positions in a Tertiary Care Setting. Witness: The Canadian \\ Journal of Critical Nursing Discourse, 3(1), pp. 73-84, https://doi.org/ 10.25071/2291-5796.79
}

\begin{abstract}
:
Minority nurses (MNs) are underrepresented in leadership roles in the Canadian healthcare system. The purpose of this study was to explore MNs' perceptions and experiences with regard to career development and MNs in leadership positions. Twelve nurses, four Caucasian and eight from the Caribbean and Africa in a tertiary care setting, were recruited through purposive sampling. Face-to-face semi-structured interviews were conducted, transcribed, coded and analyzed using critical ethnography. The findings revealed four themes: 1) social support; 2) equal opportunities; 3) lack of recognition; and 4) lack of trust. Despite negative experiences, MNs recognized the value of their work experience at the hospital where they were employed. Committing to a diverse workforce in leadership roles can ultimately have an effect on patient care. MNs' leadership is needed to provide role models for nurses and to ensure the delivery of competent care to diverse populations.
\end{abstract}

Key words: minority nurses, social capital, leadership, nurse managers, diversity, othering

\section{Introduction}

In recent decades, Canada has experienced significant demographic changes; it has developed from a predominantly White colonial society of European settlers to a multi-ethnic society composed of different cultures and traditions (Statistics Canada, 2018). This shift in population demographics requires better representation of diversity in Canadian nursing and healthcare leadership roles, such as managerial positions (Premji \& Etowa, 2012). The Canadian nursing workforce remains disproportionately Caucasian, perpetuating dominant ways of practice (Jefferies et al., 2018). The underrepresentation of minority nurses (MNs) in leadership positions is becoming an increasingly relevant topic of research as healthcare organizations strive to optimize diverse healthcare delivery to meet the needs of a diverse population. People from visible minorities (VM) are defined by the Canadian Acts of 1985 and Canada's Employment Equity Act of 1995 as "persons, other than aboriginal peoples, who are non-Caucasian in race or nonWhite in colour" (Statistics Canada, 2018, p. 1). The authors used "African Caribbean and Black nurse (ACBN)" and "minority nurse (MN)" interchangeably in this article. Over seven million Canadians identified as members of a VM group in the 2016 census, accounting for $22.3 \%$ of the total population. In the health care system, they represented $17.1 \%$ in the Montreal area and only $1 \%$ in the rest of Quebec 
(Commission des droits de la personne et des droits de la jeunesse, 2016). However, no recent statistics could be found for the rest of Canada, which makes it difficult to understand the problem and therefore justifies the importance of this study. VMs are highly visible and recognizable by the colour of their skin, facial features, and accent. These traits can be used as social markers, which lead to differentiation between social groups with the effect of marginalizing some in society (Ontario Human Rights Commission, 2009). VMs are more likely to face disparities in health care and health outcomes, which adversely affect their health and well-being; thus, reducing health inequalities is an important challenge for stakeholders (Thomas, 2014).

Nurse leaders can promote a healthier future for VM communities through health and social policies (Cope \& Murray, 2017). VM populations have distinct health needs that can be addressed through strong advisory guidance by representative nursing leaders (Phillips \& Malone, 2014). The contributions of MNs in eliminating health disparities are well documented (Phillips \& Malone, 2014; Banister $\&$ Winfrey, 2012). The career advancement of MNs cannot be extracted from social, political, and historical contexts; the need to examine the influence of race and racial ideologies on career development remains an important task. A key objective of the study was to problematize and transform misconceptions about MNs toward a more enlightened awareness of issues impacting their career advancement.

\section{State of Knowledge}

Several research studies have reported that MNs experience discrimination and underrepresentation in nursing leadership positions (Etowa et al., 2009; Jefferies et al., 2018; Premji \& Etowa, 2012). MNs experience challenges that are the result of an intersection of race and ethnicity (Zajac, 2011). Mayor (2004) found that race, class, gender, and personal phenotype shaped the career development of her participants. She therefore recommended that education and training be offered at the professional and organizational levels as a means to combat racism. Wilson (2014) contends that stereotyping, bias, and subtle internal organizational barriers prevent VMs from breaking the glass ceiling. Other researchers suggest that MNs experience discrimination, marginalization, and lack of career advancement opportunities (Iheduru-Anderson \& Wahi, 2018; Mapedzahama et al., 2011). As a result, researchers have called for recruitment and retention policies to address $\mathrm{MN}$ underrepresentation and facilitate their integration into leadership positions. This can occur through academic support, mentoring, and increasing cultural competency among their Caucasian counterparts (Covell et al., 2017; Phillips \& Malone, 2014). Alexis and Vydelingum (2004) found that MNs received limited support from their Caucasian counterparts. Nurse leaders play a major role in creating a supportive work environment, an inclusive othering where cultural differences are valued and individual differences are supported and accommodated. Canales (2010) argues that "othering" correlates with the "visibility" (e.g., skin colour, presence of an accent, sexual orientation) of one's otherness (p. 5). She contends that othering often uses power within relationships for domination and subordination, with the potential consequences being alienation, marginalization, decreased opportunities, internalized oppression, and exclusion. Ayoola (2013) reported that an inclusionary understanding of ethnic differences promotes better nurse-nurse communication and patient outcomes, whereas Villarruel et al. (2015) suggest that nursing leaders need to identify and disrupt exclusionary othering processes that undermine diversity in healthcare.

\section{Social Capital and Career Advancement}

In Canada, the prevalence of MNs in leadership positions does not mirror the racial and ethnic composition of the nation's population. Bourdieu's notion of social capital refers to all resources that one can mobilize at the social level (i.e., family network, friends, colleagues, etc.) or cultural level (habitus, education, credentials, etc.) to achieve one's personal objectives, 
particularly in terms of accessing a prestigious position (Bourdieu, 1980). Putnam (2001) distinguishes between bonding and bridging social capital: bonding is somewhat exclusive (inward looking/claustrophobic), while bridging refers to networks that are more heterogenic (outward looking), more inclusive, and inspiring tolerance.

The notion of social connection is essential in sociology: Social connections are needed to survive the vagaries of life and for the recognition of a person's existence (Bourdieu, 1986). Granovetter (1995) distinguishes between two types of relationships: those that connect a person to family and close friends (strong links) and those formed through a wider and more distant network (weak links). He notes that weak links are likely to bring more relevant information, which can lead to more satisfying and better-paid jobs.

\section{Theoretical Framework}

Othering is a process by which a person or group of persons are identified to be different from oneself or the mainstream, and it can reinforce and reproduce positions of domination and subordination. In nursing, Canales (2010) conceptualized othering as two interrelated processes: exclusionary and inclusionary. While exclusionary othering may cause alienation, marginalization, and oppression, inclusionary othering may result in coalition building, understanding, and power sharing. Othering is often influenced by the heightened visibility of the "other" brought on by their skin colour, accent, language, physical appearance, gender or age (Bouabdillah et al., 2016). The term 'othering' is used to refer to phenomena of stereotyping and racialization. The concept of othering is used to frame power relations within the organizational hierarchy of hospital settings. This concept allows a better grasp of the social construction of categories of otherness (we/them) which often emerge in the context of racialized interactions. Thus, questions of culture, race, ethnicity, and discrimination are mobilized to better understand the low representation of MNs in senior positions. The social inclusion and the critical race theory are central to the debate on MNs' underrepresentation in leadership positions. Thus, social capital is adopted as a key process in mitigating the social exclusion of disadvantaged MNs seeking career mobility. The critical race theory helped question how race subtly influences institutions' functioning and how the invisible barriers in place need to be addressed to affect change (Ford \& Airhihenbuwa, 2010). Our study investigates to what degree participants had been structured by institutionalised discrimination and stereotyping by managers and colleagues in their daily life experience.

\section{Design and Method}

Critical ethnography was chosen as the research design to examine nurses' experiences and their relationship to power and truth. It challenges us to question the construct of otherness as it applies to the professional ascension of MNs into leadership positions. Critical ethnography is an important method of inquiry that can lead to disruption in the status quo and empowerment of disenfranchised groups. The Canadian studies in healthcare settings of Baumbush (2010), Harrowing et al. (2010), and Elliott et al. (2002) are examples of the interest of Canadian researchers in critical ethnography. Another component underlying this study is the need to explore decision-making politics within an organizational culture that prioritizes a White elite. Critical ethnography also guides us to recognize the imbalances and injustices present in MNs' workplace, which disadvantage some to the benefit of others.

\section{Research Setting and Sample}

Participants were recruited from different units of a tertiary care hospital in Ontario, Canada. The hospital has a capacity of 300 beds and employs a large number of MNs from different ethnic backgrounds. Hospital administrators publicized the research by sending an email to all departments. Permission was obtained from different managers to leave information sheets about the study in their wards. Purposive sampling was used. The study sample consists of 
eight MNs and four nurse managers. All eight MNs were Black nurses from the Caribbean and Africa (ACBNs), while all the nurse managers were Caucasians; no ACBN was holding a leadership position in the organization under study. ACBNs were included if they had worked in the hospital for a year or longer, held a bachelor's degree in nursing, and held a full-time or part-time nursing position. The managers were nurses who held a bachelor's degree in nursing and who had been working as a manager for at least six months.

\section{Data Collection}

Semi-structured interviews, socio-demographic questionnaires, literature reviews, field observations, and logbooks constituted the sources of data for this study. For the observational data, a tool was developed to record relevant information, such as the nurses involved and social and professional dynamics. The main objective was to view nurses' practices and to generate a narrative-based interpretation of the events that took place. Observations took place over the course of nearly one year in different wards. The researcher was careful to remain unobtrusive and avoided sensitive or irrelevant activities. Due to ethical constraints, the P.I. observed from some distance to not hinder patient care or breach patient confidentiality. Nurses were observed during their shifts, breaks, meetings, and a seminar. A reflective journal was used as a tool to maintain reflexivity about the study and to connect the researcher's thoughts and perceptions during data analysis.

The lead author conducted all the interviews, which took place in a private meeting room at the facility and lasted approximately one hour. Participants were given time to read about the research objectives and ask questions. The interviews were audio-recorded to facilitate transcription, recall, and analysis. All participants were asked the same open question: From your work experience, what fosters or inhibits ACBNs' leadership? Other interview questions touched on participants' career experiences and whether their institution had a strategy to help talented ACBNs reach leadership positions.
Several prompts were used that allowed in-depth exploration of participants' responses (e.g., Can you give me an example? Can you tell me more about...?). When the data became redundant, it was deemed that data saturation had been reached. In total, 12 interviews were conducted.

\section{Data Analysis}

Analysis followed the first four stages of Paillé's (1994) qualitative analysis method: coding, categorizing, linking, and integrating. Interview excerpts were gathered that expressed thematic similarities, and a coding system was developed to establish relationships between these concepts (e.g., ethnicity and career advancement). Analysis of the data allowed reflection regarding the research questions and reporting on ACBNs' career advancement and promotion. Reflexivity and peer debriefing among the research team members were employed to ensure the scientific rigour of the research process and the trustworthiness of the data and analysis.

\section{Findings}

Data analysis revealed four themes: social support, equal opportunities, lack of recognition, and lack of trust.

\section{Social Support}

The professional career of ACBNs is undoubtedly enriched by the support of experienced nurse managers. Mentoring enhances ACBNs' job satisfaction, but it also ensures rapid career development, increases commitment to the institution and strengthens ties between nurse managers and ACBNs. Managers contended that hospital policy regarding career development applied to all nurses regardless of their ethnic background. One nurse manager asserted:

Fostering nurses' development is an important issue in the hospital policy. Everybody has the same chance to advance in their career depending on their experience, their education, diploma... no matter if they are Black 
or White...(Nm2)

However, an ACBN stated that the lack of support from White peers is one of the reasons ACBNs continue to be underrepresented in leadership positions. Furthermore, negative experiences are likely to be shared among ACBNs, which can discourage prospective applications for coveted senior positions:

Here, advanced positions are reserved just for the majority [White nurses]... I have friends who have been in intensive care. They did not even stay long. It seems that they were persecuted... I know that the positions where you should not go are in the emergency [department] (MN6).

ACBNs felt that, despite their credentials, they lacked the support, mentoring and training to advance in their career. According to one participant, this resulted in the uneven distribution of educated nurses along racial lines:

We have talented MNs who have their masters [degree] that were not given the chance to be trained to become a leader. On the contrary, they have worked on the floor [doing direct patient care] for years, while you cannot find any White nurse with a masters on the floor. (MN2)

ACBNs considered that having a supportive mentor increases the likelihood of being successful in occupying a leadership position:

If the person [ACBN] is assigned to a leading position, she needs a mentor to show her the tools. The mentor plays a dual role as coach and advisor. He must give [the $\mathrm{MN}$ ] both technical advice and explain how to do things. (MN6)

Lack of access to social capital was considered to limit ACBNs' chances of accessing these positions. For one participant, social capital matters for a successful career, and ACBNs are more likely to be promoted to leadership positions if they have close ties with a nurse manager:

[MNs] don't dare apply for higher position and even if they dare, they will not succeed. You need connections [with a nurse manager] to climb the ladder. There are no visible minority managers; all of them are White Canadians. (MN1)

Another participant stated:

In their [White managers] official language, they do not refuse Black nurses at the top, but in reality, there's no equity. You need to have someone at the top to support you, but since there are no $\mathrm{MN}$ leaders, we cannot advance. (MN12)

This participant also suggested that a lack of ACBNs in leadership positions hinders others' efforts to reach that goal.

\section{Equal Opportunities}

The availability of equal opportunities within an organization is an important indicator of equity. Access to opportunities includes providing ACBNs with chances to develop new competencies and leadership skills. One nurse manager explained that the failure of ACBNs in accessing leadership positions was due to their failure to act on everyday opportunities that are available to all nurses to develop their skills and to take initiative in the context of work.

Say, we want to develop a suicide prevention program. We send a memo to everyone, we ask them if they are interested in being trained on how to intervene early, but if they [MNs] do not read their emails and do not apply to participate we will not go and get them. (Nm3)

This participant does not seem to question the reasons behind ACBNs' lack of uptake of such opportunities and appears to attribute their decision mainly to a lack of motivation. Cultural 
diversity in the workplace brings differences in perceptions, beliefs, and values, especially for ACBNs who are perceived as 'other'. For example, one MN cites particular socioeconomic and cultural imperatives that impact ACBNs' disproportionately and can significantly limit their ability to take advantage of opportunities:

White nurses do not understand the reality we live in; they do not have to face the difficulties we are facing. MNs have to work double shifts, sixteen hours. They do not know that helping our people in Africa is culturally part of our real life, that if your father helped you go to school and succeed you have to help him back. (MN9)

Observations in the wards and during meetings and a conference on culture revealed the pervasiveness of White influence. For example, very few ACBNs participated in the conference:

I have been to conferences in our hospital a couple of times. Only me and another Black nurse were present. The others are all White. (MN5)

Findings showed that ACBNs were likely to perceive subtle discrimination. One participant said:

Lack of fairness exists in subtle ways; it's just you have no proof. No one is coming to tell you openly that they don't want to give you this position because you are different.... Even if you go to court you can't prove it. (MN1)

However, with regards to equal opportunities, a manager stated that all nurses have the same chances to achieve their career development:

We have people [ACBNs] who succeeded in climbing the ladder, depending on their integration, their leadership, and their interests too. One of our instructors, who was a doctor in her country of origin, went back to school, became an RN, and now she is instructor. ( $\mathrm{Nm} 4)$

One MN participant recounted this same story differently as she expressed concerns about ACBNs' lack of representation in leadership roles:

I know a nurse who was a physician in her country, she is a real informal leader, [has] a lot of experience. She applied repeatedly for a manager position but only got an instructor position. Until now, she has not succeeded in becoming a manager. (MN4)

An ACBN envisioned "positive action" as a solution to underrepresentation. She suggested that some leadership positions be specifically reserved for ACBNs, which would force at least some diversity in institutional decision-making structures:

It would be ok if one in twenty-five leadership positions be occupied by one MN... I think it would help minority nurses be represented in the hierarchy. (MN1)

\section{Lack of Recognition}

ACBNs play an important role in tackling the institution's shortage of nurses. However, some participants believe that their working conditions remain precarious insofar as their access to advanced positions remains very limited. For example, they cited the non-recognition of internationally educated nurses' diplomas and work experiences as a concern. They also believed that their working relationships with White colleagues were undermined by the prejudices resulting from ethnocultural differences and othering. One ACBN explained:

Coloured nurses and Caucasians are culturally different. We are always judged to be slow. Perhaps the rhythm of doing things is different. I can do a task one way and you do it in another way. Maybe it will take me two more 
minutes... but the work is well done in both cases. So just judging someone on their background or their look is not right. (MN3)

Another MN commented:

I want to know the reason why our diplomas are questioned, yet we have followed the same education, training, and procedures. None of us have a senior position. (MN9)

ACBN participants felt that they were subject to a devaluation process that does not recognize their professional value beyond filling the labour void of the nursing shortage. Several felt stigmatized and expressed feelings of shame. They also tended to blame themselves for the situation they were in, as one ACBN illustrated:

It's the fear of not succeeding in the leadership position that creates a feeling of loss of self-confidence, and you end up feeling ashamed of yourself. (MN6)

ACBNs perceived the non-recognition of their work as an obvious sign of discrimination and a form of racism.

We are in perpetual struggle against stereotyping; everything we do is related to the colour of our skin, not our knowledge. There are units where we feel that, yes, racism exists. (MN9)

\section{Lack of Trust}

The feeling of lack of trust was a recurring theme among participants. Feelings of distrust could create a vicious cycle in ACBNs' workplace that could both leave them out of important discussions or, on the contrary, put them under the microscope of peers' surveillance. For example, this participant explained how racial preconceptions among patient families themselves could generate exclusionary processes:

When the patient's family came for a visit, they bypassed me and checked with a White health care aid, and they ignored me, thinking the nurse is always White and the health care aid is always Black, which is hurtful and discriminating. The health care aid answered questions beyond her expertise that should be answered by a nurse; she never referred them to me. (MN3)

This participant expressed feeling excluded and othered by the patient's family and, subsequently, by the health care aid. By not referring the family to this ACBN, the health care aid consciously or unconsciously normalized racial presumptions and discrimination.

One participant stated that skin colour might be the reason for others' mistrust:

We [ACBNs] are stigmatized as persons who do not do things well. We are judged by the colour of our skin, not our experience and knowledge. (MN8)

Along with this participant, several other ACBNs perceived that judgments about the colour of their skin undermined how others viewed their competence and skills and predetermined their power relations with White peers. This had direct implications for the establishment of trust among coloured and non-coloured nurses. The presumption according to which ACBNs are 'inferior' and 'incompetent' constructs an 'other' in need of continuous surveillance and monitoring.

When you make a small mistake, it calls into question your whole competence. You're evaluated as incompetent. You become under surveillance. That makes you feel awful. For example, when a White nurse makes a big mistake, it's ok, but [if it's a] ACBN, "you forgot to give Tylenols" [a mild analgesic], it's a big deal. (MN7)

Another MN echoed this perception: 
You have to protect yourself all the time; you don't have the support of Caucasian nurses; you need to watch your back at all times. You're afraid to lose your job, your licence. (MN8)

Such feelings of being monitored by White peers appear to discourage ACBNs from competing for managerial positions, as they feel their actions and decisions will be scrutinized. In the event of a mistake, judgments would be harsher:

If you get the job, they wouldn't give you peace. You will be under constant surveillance because you took someone else's job... Now that you are supposedly manager, it's not unionized. For them [employer] it's very easy to kick you out. (MN6)

As a result, some ACBNs developed a personal resilience strategy of disaffiliation, resignation, and avoidance.

The feeling it gives me, it's just come, do your job and get out of there as soon as possible. You don't need to be invested... emotionally and whatever you do, it will never be enough. (MN8)

Despite the fact that most ACBN participants perceived living difficult working conditions, one of them, however, stated receiving the necessary support and coaching, which enabled her to obtain a position as instructor.

Many managers encouraged me... were quite supportive. They told me you're good, you can do it, you are already doing it. (MN5)

The ACBNs' perception of lack of social support, lack of equal opportunities, lack of recognition, and lack of trust, as well as the anticipation of negative performance evaluations, adversely impacted ACBNs' sense of confidence, motivation, and engagement.

\section{Discussion}

The findings from this study suggest that ACBNs were more likely to perceive biased treatment related to accessing management positions. They felt being treated differently in their daily activities at their institution in ways that resonated with othering. They felt being treated as 'other,' experiencing marginalization and exclusion. From their perspectives, nurse managers' pervasive Whiteness and their professional experiences obscured their understanding of the need to empower ACBNs through social capital, recognition, and trust. ACBNs expressed concerns that the balance of power favoured the White majority, thus confining them to lower socio-professional positions. Similar results were reported in an Australian study in which Hawthorne (2006) found that MNs from non-English-speaking countries were less likely to advance in their careers and were disproportionately represented in less prominent specialties, such as geriatrics. In another Australian study a few years later, Mapedzahama et al. (2011) critically examined the discourses of inclusive, non-racialized nursing in Australia. In that study, Black nurses perceived that their skin colour racially predetermined their encounters with their White peers, thus undermining the recognition of their skills, competence, and professional status. These nurses were also aware of the subtle but widespread social stereotypes that characterized everyday racism. The findings of our study corroborate the results of the aforementioned research and provide interesting new perspectives within a Canadian context. Larsen (2007) argues that surveillance, which was described by one of the participants, constitutes a form of "victimization through critical scrutiny," and that the non-recognition of MNs' skills embodies discrimination. MNs can therefore expect to shed their identity as 'others' only when the oppression perpetuated by the in-group is eliminated.

These conclusions are consistent with the observations and the statements of the participants in our research. ACBNs felt excluded from positions of power and reported receiving little mentoring or training to improve access to 
leadership positions. Using the social ecological model, Carr et al. (2017) found that mentoring was unfunded in institutions, and policies rarely prioritized it for the promotion to leadership positions for ethnic minorities. Thus, there is a missed opportunity for institutional effort to support ethnic/racial equity. Given the lack of social support from their White colleagues, ACBNs felt marginalized, excluded, and discriminated against. Since nurse managers fail to support the creation of structures of inclusion that recognize and accommodate the different realities of $\mathrm{ACBN}$, there is little chance that the institution will address the issue and that change will occur. The fact that nurse managers in this study were all Caucasian certainly plays a role in this, which further confirms the need for more diversity in executive leadership positions. Bessent (2002), an African American scholar, stresses the need for more $\mathrm{MNs}$ in leadership positions. She asserts that even with the growing number of VMs in the population, nursing is not reflective of that increase because of the few ACBNs in the profession. In light of demographic changes, the underrepresentation of ACBNs in leadership positions could directly affect the delivery of health care to minority patients since leadership diversity enhances culturally competent health care (Wilson, 2014). Racial, ethnic, and cultural diversity is a Canadian core value that must be woven throughout nursing (Etowa et al., 2011). The presence of ACBNs in leadership positions may influence organizational goals and priorities and may also influence future minority students to join the profession (Georges, 2012). Competition for senior positions tends to favour Caucasian nurses as a result of a lack of tolerance for diversity (Berry, 2010). The fact that no ACBN occupies a senior position in the institution suggests that ACBNs' low social capital may keep them in subordinate positions unless a policy of positive discrimination is upheld. Such policies, known as affirmative action in the United States, have been taken into consideration in the admission process to bolster minorities' chances to access American universities. This practice has been fiercely opposed by the 'majority' (White, Asian American), some of whom have sued universities alleging they were discriminated against in violation of the Equal Protection Clause (Hirschman et al., 2016). ACBNs' skills and ability to influence and inspire colleagues need to be empowered to strengthen their support networks. The study fits within the existing Srivastava (2007) reference framework regarding strengthening nursing practice through leadership, mentorship and technology. Another component underlying the study is the need to explore decision-making politics within an organizational culture that prioritizes a White elite. It contributes to an understanding of the career development of ACBNs. Health organizations need to hire, retain, and help these qualified nurses advance in leadership positions to meet healthcare disparities and patient safety concerns. This study presents an understanding of the career advancement of ACBNs. While working toward securing leadership roles, they face a myriad of barriers and challenges resulting in invisibility, including unequal advancement opportunities, lack of leadership preparation and mentoring. ACBN role models are needed to facilitate the career development of nurses.

Concerning education, the authors urge leading managers to provide key resources to ACBNs and understand how to foster bridging social capital to build diversity and cohesiveness. They have the responsibility to exchange knowledge and mentor these nurses by encouraging them to use their creativity and skills and guide them in future leadership duties.

There is a need for future studies and updated statistics to determine the state of the issue, the trends regarding ACBN integration and mentoring initiatives. Furthermore, our study sample did not include any Canadian-born MNs. Perhaps their views would differ from those of ACBNs born abroad. Further research is needed to differentiate and nuance the possibly differing experiences of MNs who work in Canadian healthcare settings and delineate the factors and contingencies that produce such differences. Finally, notions such as social capital and othering constitute important concepts that can help better understand ACBNs' continued underrepresentation in leadership positions in healthcare, yet they remain underutilized in 
current analyses. Further studies grounded in these concepts could help revitalize nursing research in this area.

\section{Limitations}

A key limitation for this study was the availability of statistics on $\mathrm{MN}$ representation in the Canadian health care system. The limited number of MNs might compromise the conclusion drawn from the study. Interviewing a Caucasian manager might have brought a different perspective to the findings. None of the managers are an $\mathrm{MN}$; therefore, we cannot identify the strategies adopted by MNs to reach a leadership position.

\section{Conclusion}

The present article generates awareness that social capital matters for MNs' career success. MNs can be empowered to play a significant role in influencing their institution's performance in a positive way. This study identified several key elements that may help nurse managers and institutions supporting exclusionary policies based on White privilege to identify and disrupt exclusionary practices by including $\mathrm{MNs}$ in mentoring programs.

The goal of the study was to explore the underrepresentation of MNs in leadership roles and the factors that might hinder their career development. The study shows that MNs are underrepresented at the leadership level and that individual and structural factors are at play to explain this. MNs' experiences suggest they are 'othered', meaning that they felt discriminated against, experienced stigma, and were distrusted.

\section{References}

Alexis, O. \& Vydelingum V. (2004). The lived experiences of overseas black and minority ethnic nurses in the National Health Service in the south of England. Diversity Health Social Care, 1(1), 13-20.
These issues are unlikely to be remedied if, as our findings show, managers feel that current mentoring and training programs are sufficient to promote career advancement for MNs. From MNs' perspectives, nurse managers' pervasive Whiteness and their professional experiences lack empowering leadership through mentoring, recognition, and supporting $\mathrm{MN}$ development, which can create a trusting relationship. Nurses' social capital in their work environment may be enhanced by improving communication, understanding, trust, and positive leadership practices and policies, which could result in positive relationships between nurses, managers, and the overall institution. The institution faces the challenge of providing equal opportunity employment and fair hiring practices. The institution needs to empower MNs through education and other sources of internal and external support. The limited number of MNs to serve as role models and mentors creates an additional barrier to the successful recruitment and proper representation of minority nurses.

\section{Ethical Permission}

Ethics approval for this research was obtained from the hospital and from the Office of Research Ethics and Integrity, University of Ottawa.

\section{Declaration of Conflicting Interests}

The author(s) declare no potential conflicts of interest with respect to the research, authorship and publication of this article.

\section{Funding}

The study was supported with funding from the host hospital, who is protected by a confidentiality agreement stipulated in the informed consents.

Ayoola, A. (2013, May 7). Why diversity in the nursing workforce matters. Robert Wood Johnson Foundation. https://www.rwjf.org/en/blog/2013/05/why _diversity_inthe.html

Banister, G., \& Winfrey, M. E. (2012). Enhancing diversity in nursing: A partnership approach. JONA: The Journal of Nursing Administration, 42(3), 176-181. 
doi:10.1097/nna.0b013e3182480a97

Baumbush, J. (2010). Conducting critical ethnography in long-term residential care. Journal of Advanced Nursing, 67(1), 184192. doi:10.1111/j.1365-2648.2010.05413.x

Berry L.E. (2010) Making a diversity difference: stories of leadership in creating a more inclusive nursing profession (doctoral dissertation). University of Saskatchewan, Saskatchewan, Canada.

Bessent, H. (2002). Minority nurses in the new century. Characteristics and workforce utilization patterns: A survey. Washington, DC: American Nurses Association.

Bouabdillah, N., Holmes, D., \& Tourigny, J. (2016). Infirmières issues de minorités visibles et mobilité verticale en milieu hospitalier. Recherche en soins infirmiers, 127(4), 71-81. doi:10.3917/rsi.127.0071

Bourdieu, P. (1980). Le capital social, notes provisoires. Actes de la recherche en sciences sociales, 31, 2-4.

Bourdieu, P. (1986). The forms of capital. In J. Richardson (Ed.), Handbook of theory and research for the sociology of education (pp. 241-258). Greenwood.

Canales M. K. (2010). Othering: Difference understood?: A 10-year analysis and critique of the nursing literature. Advances in Nursing Science, 33, 15-34. doi:10.1097/ans.0b013e3181c9e119

Carr, P. L., Gunn, C., Raj, A., Kaplan, S., \& Freund, K. M. (2017). Recruitment, promotion, and retention of women in academic medicine: how institutions are addressing gender disparities. Women's Health Issues: Official Publication of the Jacobs Institute of Women's Health, 27(3), 374-381. doi:10.1016/j.whi.2016.11.003

Commission des droits de la personne et des droits de la jeunesse, Rapport triennal 2013-
2016, Loi sur l'accès à l'égalité en emploi dans des organismes publics, décembre 2016, pp. 20-39.

Cope, V., \& Murray, M. (2017). Leadership styles in nursing. Nursing Standard, 31(43), 61-70. doi:10.7748/ns.2017.e10836

Covell, C., Primeau, M., Kilpatrick, K., \& StPierre, I. (2017). Internationally educated nurses in Canada: Predictors of workforce integration. Human Resources for Health, 15(1), 26. doi:10.1186/s12960-017-0201-8

Elliott, J., Berman, H., \& Kim, S. (2002). A critical ethnography of Korean Canadian women's menopause experience. Health Care for Women International, 22, 377-388. doi:10.1080/0739933029008946

Etowa, J. B., Price, S., \& Debs-Ivall, S. (2011). Strengthening the ethno-cultural diversity of the nursing workforce in Canada.

International Journal of Arts \& Sciences, 4(26), 75-87.

Etowa, J. B., Sethi, S., \& Thompson-Isherwood, R. (2009). The substantive theory of surviving on the margin of a profession. Nursing Science Quarterly, 22(2), 174-181. doi:10.1177/0894318409332781

Ford, C. L., \& Airhihenbuwa, C. O. (2010). Critical race theory, race equity, and public health: Toward antiracism praxis. American Journal of Public Health, 100(S1). 30-35. doi:10.2105/ajph.2009.171058

Georges, C. A. (2012). Project to expand diversity in the nursing workforce. Nursing Management, 19(2), 22-26. doi:10.7748/nm2012.05.19.2.22.c9061

Granovetter, M. (1995). Getting a Job: A Study of Contacts and Careers (2nd ed.). University of Chicago Press.

Harrowing, J. N., Mill, J., Spiers, J., Kulig, J., \& Kipp, W. (2010). Culture, context, and community: Ethical considerations for global nursing research. International Nursing Review, 57, 70-77. 
doi:10.1111/j.1466-7657.2009.00766.x

Hawthorne, L. (2006). Labour market outcomes for migrant professionals: Canada and Australia compared-executive summary. Citizenship and Immigration Canada, Ottawa.

Hirschman, D., Berrey, E., \& Rose-Greenland, F. (2016). Dequantifying diversity: Affirmative action and admissions at the University of Michigan. Theory and Society, 45, 265-301. doi:10.1007/s11186-016-9270-2

Iheduru-Anderson, K. C., \& Wahi, M. M. (2018). Experiences of Nigerian internationally educated nurses transitioning to United States health care settings. Journal of Transcultural Nursing, 29(6), 603-610. doi: $10.1177 / 1043659618766225$

Jefferies, K., Tamlyn, D., Aston, M., \& Tomblin Murphy, G. (2018). Promoting visible minority diversity in Canadian nursing. Canadian Journal of Nursing Research, 51(1), 3-5. doi: $10.1177 / 0844562118795812$

Larsen, J. A. (2007). Embodiment of discrimination and overseas nurses' career progression. Journal of Clinical Nursing, 16, 2187-2195. doi:10.1111/j.1365-2702.2007.02017.x

Mapedzahama, V., Rudge, T., Westa, S., Perron, A., (2011). Black nurse in White space? Rethinking the in/visibility of race within the Australian nursing workplace. Nursing Inquiry, 19(2), 153-164. doi:10.1111/j.1440-1800.2011.00556.x

Mayor, V. (2004). Climbing high: A look at strategies used by leading Black nurses in their career development. Nursing Standard, 18(34), 104. doi:10.7748/ns2004.05.18.34.104.c3603

Ontario Human Rights Commission. Policy and Guidelines on Racism and Racial Discrimination (approved June 9, 2005). http://www.ohrc.on.ca
Paillé, P. (1994). L'analyse par théorisation ancrée. Cahiers de Recherche Sociologique, 23, 147-181.

Phillips, J. M., \& Malone, B. (2014). Increasing racial/ethnic diversity in nursing to reduce health disparities and achieve health equity. Public Health Reports, 129(1_suppl2), 4550.

doi:10.1177/00333549141291s209

Premji, S., \& Etowa, J. B. (2012). Workforce utilization of visible and linguistic minorities in Canadian nursing. Journal of Nursing Management, 22(1), 80-88. doi:10.1111/j.1365-2834.2012.01442.x

Putnam, R. D. (2001). Bowling alone: The collapse and revival of American community. Simon and Schuster.

Srivastava R. (2007). The Healthcare Professional's Guide to Clinical Cultural Competence. Elsevier, Toronto, ON.

Statistics Canada, (2018). Census Profile, 2016 (Census. Statistics Canada Catalogue No. 98-316- X2016001). Ottawa, ON.

Thomas, B. (2014), Health and health care disparities: The effect of social and environmental factors on individual and population health. International Journal of Environmental Research Public Health, 11, 7492-7507. doi:10.3390/ijerph110707492

Villarruel, A., Washington, D., Lecher, W. T., Carver, N. A. (2015). A more diverse nursing workforce: Greater diversity is good for the country's health. American Journal of Nursing, 115(5), 57-62. doi:10.1097/01.naj.0000465034.43341.b1

Wilson, E. (2014). Diversity, culture and the glass ceiling. Journal of Cultural Diversity, 21(3), 83-89.

Zajac, L. (2011). Double-loop approach: Recruitment and retention of minority nursing faculty. ABNF Journal, 22(3). 